\title{
A NOTE ON SOME ANALYTIC AND GEOMETRIC PROPERTIES OF CERTAIN MULTIVALENT FUNCTIONS
}

\author{
H. IRMAK
}

ABSTRACT. In consideration of fractional calculus and integral operator, some complex equations and/or inequalities specified by certain multivalently analytic functions in the unit open disk are first presented and then certain consequences in relation with analytic and geometric properties of certain multivalent functions are pointed out.

2010 Mathematics Subject Classification: 26A33, 33E12, 30C80, 26E35, 30C45.

Keywords: Unit open disk, complex plane, multivalent, analytic, fractional calculus, equations and inequalities in the complex plane, analytic and geometric properties.

\section{Introduction, Definitions and Preliminaries}

It is well known truth that fractional calculus is both an important branch of classical mathematics, which deals with the generalization of operations of differentiation and integration to fractional order, and a comprehensive research field of mathematical analysis which may be taken for an old as well as a modern topic. Fractional calculus is an old topic because of its long history starting from some notes and ideas of G. W. Leibniz and L. Euler. On the other hand, it is a modern topic due to its enormous development during the last two decades. As is known, the present interest of many scientists and engineers in the theory of fractional calculus has been initiated by applications of this theory as well as by new mathematical challenges. In the same breath, fractional differential equations (or, also known as extraordinary differential equations) are obtained by generalizing differential equations to an arbitrary order. Since fractional differential equations are used to model complex phenomena, they also play a crucial role in engineering, physics and applied mathematics. Therefore, they have been generating increasing interest from engineers and scientist in recent years. Since fractional differential equations have memory, non-local relations in space and time, complex phenomena can be modeled by using 
these equations. Due to this fact, materials with memory and hereditary effects, fluid flow, rheology, diffusive transport, electrical networks, electromagnetic theory and probability, signal processing, and many other physical processes are diverse applications of fractional differential equations.

The object with this work is to present an investigation relating to some comprehensive results between certain types of complex fractional (differential) equations and complex functions with one variable, which are (multivalently) analytic functions in certain domains of complex plane, and to reveal some analytical and geometrical properties of them. We also note that our results include a number of the earlier results given in [6]. For our purpose, we need to introduce certain notations and definitions, and also to recall a few well known assertions.

Firstly, here and throughout this paper, let $\mathbb{R}$ be the set of real numbers, let $\mathbb{C}$ be the set of complex numbers, let $\mathbb{N}$ be the set of positive integers, let $\mathbb{U}$ be the open unit disk, that is, that the set $\mathbb{U}:=\{z \in \mathbb{C}:|z|<1\}$.

Let also $\mathcal{H}(\mathbb{U}), \mathcal{H}_{p}$ and $\mathcal{A}_{p}$ be the families of all functions which are analytic in the disk $\mathbb{U}$, the family of analytic functions in the following forms:

$$
\mathcal{H}_{p}=\left\{f \in \mathcal{H}(\mathbb{U}): f(z)=p+\sum_{n=p}^{\infty} b_{n} z^{n} \quad(z \in \mathbb{U})\right\}
$$

and the family of multivalently analytic functions in the following forms:

$$
\mathcal{A}_{p}=\left\{f \in \mathcal{H}(\mathbb{U}): f(z)=z^{p}+\sum_{n=p+1}^{\infty} b_{n} z^{n} \quad(z \in \mathbb{U})\right\},
$$

respectively. Additionally, let $\mathcal{H}:=\mathcal{H}_{1}$ and $\mathbb{R}^{*}:=\mathbb{R} \backslash\{0\}$. It is clear that $\mathcal{H}_{p} \subset \mathcal{H}(\mathbb{U})$ and $\mathcal{A}_{p} \subset \mathcal{H}(\mathbb{U})$.

For the parameter $\gamma(\gamma>0)$ and an analytic function $f(z)$ in $\mathcal{A}_{p}$, we want to recall the following integral operator:

$$
\mathcal{P}^{\gamma}[f]:=\mathcal{P}^{\gamma}[f(z)]=\frac{(p+1)^{\gamma}}{z \Gamma(z)} \int_{0}^{z}\left[\log \left(\frac{z}{\kappa}\right)\right]^{\gamma-1} f(\kappa) d \kappa
$$

where the function $\Gamma$ is well-known gamma function.

By applying the operator given by (3) to a function $f \in \mathcal{A}_{p}$, we can easy to determine that

$$
\mathcal{P}^{\gamma}[f]=z^{p}+\sum_{n=p+1}^{\infty}\left(\frac{p+1}{n+1}\right)^{\gamma} a_{n} z^{n} \quad(\gamma>0) .
$$


For the details of the operator and also some of its applications, one may center on the results or the works in the references in [6], [11] and [14]. In view of (4) and also after certain calculations, the following identity in relation with the operator given by (3):

$$
z \frac{d}{d z}\left(\mathcal{P}^{\gamma}[f]\right) \equiv z\left(\mathcal{P}^{\gamma}[f]\right)^{\prime}=(p+1) \mathcal{P}^{\gamma-1}[f]-\mathcal{P}^{\gamma}[f]
$$

can be easily obtained, where $\gamma>0$ and $f \in \mathcal{A}_{p}$.

Next, the most frequently encountered tools in the theory of fractional calculus (that is, differentiation and integration of an arbitrary real or complex order) are presented by the familiar differ-integral operator ${ }_{c} \mathcal{D}_{z}^{\mu}(\cdot)$, defined by

$$
{ }_{c} \mathcal{D}_{z}^{\mu}(f(z)):=\left\{\begin{array}{cl}
\frac{1}{\Gamma(-\mu)} \int_{c}^{z} \frac{f(\tau)}{(z-\tau)^{1-\mu}} d \tau & (c \in \mathbb{R} ; \Re e(\mu)<0) \\
\frac{d^{m}}{d z^{m}}\left({ }_{c} \mathcal{D}_{z}^{\mu-m}(f(z))\right) & (m-1 \leq \Re e(\mu)<m ; m \in \mathbb{N}),
\end{array}\right.
$$

provided that the integral exists. For $c:=0$, the operator $\mathcal{D}_{z}^{\mu}(\cdot)$ given by

$$
\mathcal{D}_{z}^{\mu}(f(z)):={ }_{0} \mathcal{D}_{z}^{\mu}(f(z)) \quad(\mu \in \mathbb{C})
$$

corresponds essentially to the classical (Riemann-Liouville) fractional derivative (or integral) of order $\mu$ (or $-\mu$ ). In special, for the function $f(z)=z^{\kappa}$, of course, with $c:=0$, it is easily seen that

$$
\mathcal{D}_{z}^{\mu}\left(z^{\kappa}\right):=\frac{\Gamma(\kappa+1)}{\Gamma(\kappa-\mu+1)} z^{\kappa-\mu} \quad(\Re e(\kappa)>-1) .
$$

For further details of the related definitions and their applications, and also for some results relating to both certain fractional (differential) equations and multivalently analytic functions, one may refer to the works [1], [6], [7], [8], [9], [10] and also [13].

For the main result, there is a need to recall the following well-known assertion, which is Lemma 1 and also obtained by [12].

Lemma 1. Let $q(z)$ be in the class $\mathcal{H}$. If there exists a point $z_{0}$ in $\mathbb{U}$ such that

$$
\Re e(q(z))>0\left(|z|<\left|z_{0}\right|\right), \Re e\left(q\left(z_{0}\right)\right)=0 \text { and } q\left(z_{0}\right) \neq 0
$$

then

$$
\left.q(z)\right|_{z=z_{0}}=i a \quad \text { and }\left.\quad z q^{\prime}(z)\right|_{z=z_{0}}=\left.q(z)\right|_{z=z_{0}} i \kappa\left(a+\frac{1}{a}\right),
$$

where $\kappa \geq \frac{1}{2}$ and $a \in \mathbb{R}^{*}$. 
H. Irmak - Some properties of certain multivalent functions ...

\section{An Extensive Result And its Proof}

We begin by setting and then by proving the following result consisting of certain equations or inequalities in the complex plane.

Theorem 1. Let a function $\Phi(z)$ in the class $\mathcal{H}(\mathbb{U})$ satisfy any one of the cases of the following inequality:

$$
\Re e(\Phi(z))>\left\{\begin{array}{cll}
\frac{\alpha}{2(\alpha-p)} & \text { if } 0 \leq \alpha \leq \frac{p}{2} \\
\frac{\alpha-p}{2 \alpha} & \text { if } \frac{p}{2} \leq \lambda<p
\end{array} \quad(p \in \mathbb{N} ; z \in \mathbb{U}) .\right.
$$

If a function $f(z)$ belonging to the class $\mathcal{A}_{p}$ is a solution for the following complex type fractional equation:

$$
z \mathcal{D}_{z}^{1+\mu}\left(\mathcal{P}^{\gamma}[f]\right)+(\mu-p-\Phi(z))\left(\mathcal{D}_{z}^{\mu}\left(\mathcal{P}^{\gamma}[f]\right)\right)=0
$$

then

$$
\Re e\left(\frac{\mathcal{D}_{z}^{\mu}\left(\mathcal{P}^{\gamma}[f]\right)}{z^{p-\mu}}\right)>\frac{\alpha \Gamma(p)}{\Gamma(p-\mu+1)},
$$

where $\gamma>0,0 \leq \alpha<p, 0 \leq \mu<1, p \in \mathbb{N}$ and $z \in \mathbb{U}$.

Proof. In consideration of (2), (4), and (6) and also after some elementary calculations, one can easily determine:

$$
\mathcal{D}_{z}^{\mu}\left(\mathcal{P}^{\gamma}[f]\right)=\frac{\Gamma(p+1)}{\Gamma(p-\mu+1)}\left(1+\sum_{n=1}^{\infty} \Omega_{n}(p, \mu ; \gamma) a_{n+p} z^{n-p}\right) z^{p-\mu}
$$

Next, define an implicit function $q(z)$ by

$$
\begin{aligned}
\mathcal{D}_{z}^{\mu}\left(\mathcal{P}^{\gamma}[f]\right) & =\left(1+\sum_{n=1}^{\infty} \Omega_{n}(p, \mu ; \gamma) a_{n+p} z^{n}\right) \frac{\Gamma(p+1)}{\Gamma(p-\mu+1)} z^{p-\mu} \\
& =\left[\frac{\alpha}{p}+\left(1-\frac{\alpha}{p}\right) q(z)\right] \frac{\Gamma(p+1)}{\Gamma(p-\mu+1)} z^{p-\mu},
\end{aligned}
$$

where

$$
\gamma>0,0 \leq \mu<1,0 \leq \alpha<p, n \in \mathbb{N}, p \in \mathbb{N}
$$

and

$$
\Omega_{n}(p, \mu ; \gamma):=\frac{\Gamma(n+1) \Gamma(p-\mu+1)}{\Gamma(n-\mu+1) \Gamma(p+1)}\left(\frac{p+1}{n+p+1}\right)^{\gamma} .
$$


H. Irmak - Some properties of certain multivalent functions ...

It is obvious that $q(z)$ is a member of the class $\mathcal{H}$. In short, $q(z)$ is an analytic function in $\mathbb{U}$ and $q(0)=1$. By means of (9) together with (5), it is easily established that

$$
\frac{z\left[\frac{\Gamma(p-\mu+1)}{\Gamma(p-\mu+1)} z^{\mu-p} \mathcal{D}_{z}^{\mu}\left(\mathcal{P}^{\gamma}[f]\right)\right]^{\prime}}{\frac{\Gamma(p-\mu+1)}{\Gamma(p-\mu+1)} z^{\mu-p} \mathcal{D}_{z}^{\mu}\left(\mathcal{P}^{\gamma}[f]\right)}=\mu-p+\frac{z \mathcal{D}_{z}^{1+\mu}\left(\mathcal{P}^{\gamma}[f]\right)}{\mathcal{D}_{z}^{\mu}\left(\mathcal{P}^{\gamma}[f]\right)} .
$$

By taking into account (10), it follows from (12) that

$$
\left.\mu-p+\frac{z \mathcal{D}_{z}^{1+\mu}\left(\mathcal{P}^{\gamma}[f]\right)}{\mathcal{D}_{z}^{\mu}\left(\mathcal{P}^{\gamma}[f]\right)}=\frac{\left(1-\frac{\alpha}{p}\right) z q^{\prime}(z)}{\frac{\alpha}{p}+\left(1-\frac{\alpha}{p}\right) q(z)}(=\Phi(z)) . \quad \text { (say }\right)
$$

In view of (12), clearly, the complex function $\Phi(z)$ satisfies the fractional equation given by (7).

Supposing now that there exists a point $z_{0}$ in $\mathbb{U}$ such that

$$
\Re e(q(z))>0 \quad\left(|z|<\left|z_{0}\right|\right), \Re e\left(q\left(z_{0}\right)\right)=0 \text { and } q\left(z_{0}\right) \neq 0 .
$$

By applying the assertions of Lemma 1, which are

$$
q\left(z_{0}\right)=i a \quad \text { and }\left.\quad \frac{z q^{\prime}(z)}{q(z)}\right|_{z=z_{0}}=i c\left(a+\frac{1}{a}\right) \quad\left(c \geq \frac{1}{2} ; a \in \mathbb{R}^{*}\right)
$$

and using the following inequalities:

$$
0 \leq \alpha \leq \frac{p}{2} \Longrightarrow \frac{1+a^{2}}{a^{2}+\left(\frac{\alpha / p}{1-\alpha / p}\right)^{2}} \geq 1
$$

and

$$
\frac{p}{2} \leq \alpha<p \quad \Longrightarrow \quad \frac{1+a^{2}}{1+a^{2}\left(\frac{1-\alpha / p}{\alpha / p}\right)^{2}} \geq 1,
$$

respectively, then (13) yields that

$$
\begin{aligned}
\Re e\left(\Phi\left(z_{0}\right)\right) & =\frac{\frac{c \alpha}{p}\left(\frac{\alpha}{p}-1\right)\left(1+a^{2}\right)}{\left(\frac{\alpha}{p}\right)^{2}+a^{2}\left(1-\frac{\alpha}{p}\right)^{2}} \\
& \leq \frac{\frac{\alpha}{p}\left(\frac{\alpha}{p}-1\right)\left(1+a^{2}\right)}{2\left[\left(\frac{\alpha}{p}\right)^{2}+a^{2}\left(1-\frac{\alpha}{p}\right)^{2}\right]}
\end{aligned}
$$


H. Irmak - Some properties of certain multivalent functions ...

$$
\leq\left\{\begin{array}{cll}
\frac{\alpha}{2(\alpha-p)} & \text { for } & 0 \leq \alpha \leq \frac{p}{2} \\
\frac{\alpha-p}{2 \alpha} & \text { for } & \frac{p}{2} \leq \lambda<p
\end{array},\right.
$$

since $c \geq 1 / 2$ and $\alpha / p-1<0$. It is quite obvious that the inequalities given by (14) are contradictions with the assumptions given by (8), respectively. Hence, the statement, given by (10), yields the the inequality in (9). Therefore, the desired proof is completed.

\section{A few Analytic and Geometric Consequences}

By taking aim at the main result, namely, Theorem 1, it is easy seen that there are several comprehensive applications of theorem which has important role in the theories of analytic functions and (certain types of) complex (fractional type differential) equations, as consequences of the main result. In special, some of the consequences of Theorem 1 are important results for the theory of analytic and geometric functions (see, [2] and [4], and see, for example, [5], [10] and [9]). For both these and the other possible consequences of Theorem 1, it is enough to choose appropriate values of the parameter(s) in the related theorem. Of course, it is not possible to determine all of them. But, particularly, as example, we want to focus on only three consequences of them, which deal with an interesting results consisting of both certain complex (fractional type) differential equations and multivalently analytic functions, which are below. The other possible consequences of all theorem (which are here omitted) are presented to the attention of the researchers who have been working on the theories of (complex) differential equations and/or (multivalently) analytic functions in certain domains of the complex plane.

Firstly, by choosing suitable values of the parameter $\mu$ (and also by taking into consideration the well known identity given by (5)), the two-earlier results, which obtained by [6] and also include (both analytic and) geometric properties are obtained by the following remarks.

Remark 1. In view of (5), by taking $\mu:=0$ in Theorem 1, the result obtained by [[6], Theorem 2.1], which also includes multivalently close-to-starlikeness of order $\alpha / p(0 \leq \alpha<p ; p \in \mathbb{N})$, is then achieved.

Remark 2. By taking $\mu \rightarrow 1-$ in Theorem 1, the result obtained by [6, Theorem 2.2], which also includes multivalently close-to-convexity of order $\alpha(0 \leq \alpha<p$; $p \in$ $\mathbb{N})$, are also attained.

Lastly, by letting $\gamma \rightarrow 0^{+}$in Theorem 1 , the following result is established by corollary 1. 
H. Irmak - Some properties of certain multivalent functions ...

Corollary 1. Let the function $\Psi(z)$ be in the class $\mathcal{A}_{p}$ and also satisfy any one of the cases of the inequalities given by (7). If a function $w:=f(z)$ belonging to the class $\mathcal{A}_{p}$ is a solution for the following complex type equation given by

$$
z \mathcal{D}^{1+\mu}[w]+(\mu-\Psi(z)-p) \mathcal{D}^{\mu}[w]=0
$$

then

$$
\Re e\left(z^{\mu-p} \mathcal{D}^{\mu}[w]\right)>\frac{\alpha \Gamma(p)}{\Gamma(p-\mu+1)},
$$

where $0 \leq \mu<1,0 \leq \mu<p, p \in \mathbb{N}$ and $z \in \mathbb{U}$

\section{REFERENCES}

[1] M.P. Chen, H. Irmak, H.M. Srivastava, A certain subclass of analytic functions involving operators of fractional calculus, Comput. Math. Appl. 35, 2 (1998), 83-91.

[2] P.L. Duren, Grundlehren der Mathematischen Wissenchaffen. Springer-Verlag, New York, Berlin, Heidelberg, Tokyo, 1983.

[3] S. Fukui, K. Sakaguchi, An extension of a theorem of S. Ruscheweyh, Bull. Fac. Ed. Wakayama Univ. Natur. Sci. 29, (1980), 1-3.

[4] A.W. Goodman, Univalent functions. Vols. I and II., Polygonal Publishing House, Washington-New Jersey, 1983.

[5] H. Irmak, Some novel applications in relation with certain equations and inequalities in the complex plane, Math. Commun. 23, 1 (2018), 9-14.

[6] H. Irmak, Certain complex equations created by integral operator and some of their application to analytic functions, Le Math. 71, 1 (2016), 43-49.

[7] H. Irmak, R.K. Raina, Some applications of generalized fractional calculus operators to a novel class of analytic functions with negative coefficients, Taiwanese J. Math. 8, 3 (2004), 443-452.

[8] H. Irmak, A few inequalities established by using fractional calculus and their applications to certain multivalently analytic functions, Chin. J. Math. (New York) 2014, Art. ID 349719, 5 pp.

[9] H. Irmak, N. Tuneski, Fractional calculus operator and certain applications in geometric function theory, Sarajevo J. Math. 6, 18 (1) (2010), 51-57.

[10] M. Şan, H. Irmak, Some novel applications of certain higher order ordinary complex differential equations to normalized analytic functions, J. Appl. Anal. Comput. 5, 3 (2015), 474-484. 
H. Irmak - Some properties of certain multivalent functions ...

[11] I. B. Jung, Y. C. Kim, H. M. Srivastava, The Hardy space of analytic functions associated with certain one-parameter families of integral operators, J. Math. Anal. Appl. 176, 1 (1993), 138-147.

[12] M. Nunokawa, On properties of non-Caratheodory functions, Proc. Japan Acad. Ser. A Math. Sci. 68, (1998), 152-153.

[13] S.G. Samko, A.A. Kilbas and O. I. Marichev, Fractional Integrals and Derivatives: Theory and Applications. Gordon and Breach Science Publishers, Yverdon, Switzerland, 1993.

[14] S. Shams, S. R. Kulkarni and J. M. Jahangiri, Subordination properties of pvalent functions defined by integral operators, Inter. J. Math. and Math. Sci. 2006, Article ID 94572, 3 pages.

Hüseyin IRMAK

Department of Mathematics

Faculty of Science

Çankırı Karatekin University

Uluyazı Campus

Tr-18100, Çankırı

TURKEY

e-mail: hisimya@yahoo.com or hirmak@karatekin.edu.tr 